\title{
Indications for Digital Fluorography and Storage-Phosphor Plates in Pediatrics: Certainties and Questionable Points
}

\author{
Ch. Durand, Ph. Baudain, P. Francois, and M. Kandelman
}

\begin{abstract}
We have used two digital imaging systems: digital fluorography and the phosphor plates system. The respective indications for each technique in pediatric practice are presented hereafter. Digital fluorography is highly recommended for dynamic examinations, and phosphor plates are highly recommended for conventional studies, if the spatial resolution is mandatory. Either digital fluorography or phosphor plates can be used for contrast studies. In pediatric radiology, if the spatial resolution is not mandatory, digital fluorography is suggested to reduce the radiation dose, and if using a small fields of view is possible, digital fluorography may be carefully discussed. Digital radiography is supported neither to be better than classical radiology nor to compensate for technician's mistakes. It is aimed to improve the department's functioning and, above all, to accommodate future innovations. Images can be transferred or archived through networks. This is a main prospect in the coming years.

Copyright $\odot 1995$ by W.B. Saunders Company
\end{abstract}

KEY WORDS: digital fluorography, phosphor plates system, pediatric radiology.

$\mathbf{I}^{\mathrm{N}}$ N THE PEDIATRIC RADIOLOGY Department in Grenoble, we have used two digital imaging systems: digital fluorography (Digital Recording System, DRS; General Electric Medical Systems, Milwaukee, WI) for more than 4 years, and the phosphor plates system FCR $\mathrm{AC} 1+$ (Fuji, Tokyo, Japan) for three years. The characteristics of each technique are well known. We discuss the respective indications for each technique in pediatric practice.

Digital fluorography allows real-time study with immediate viewing of the images. It also allows dynamic studies, reduction of the radiation dose and fluoroscopy. It has the disadvantage of being a heavy technique, linked to a remote-controlled table. Therefore, it is not possible to perform bedside radiographs. Moreover, the field of view is reduced to approximately $30 \mathrm{~cm}$ and spatial resolution is poor.

With phosphostimulable plates, portable radiographs are possible, the field of view is not limited and spatial resolution is excellent.

It is then interesting to observe that the advantages of one method are limitations of the other and vice versa. This clearly explains why the two methods are not competing but complementary and why the indications are different.
It is the radiologist's role to determine before examination which technique will be used.

\section{INDICATIONS}

To simplify, the following situations may be encountered in current practice: dynamic studies, conventional examinations without contrast, conventional examinations with contrast, and some particular situations such as bedside radiographs or interventional radiology. Pediatric radiology has specific indications.

\section{DYNAMIC EXAMINATIONS}

Digital fluorography is the only method that realizes sequential studies. Dynamic sequential studies up to 6 views/s replace the classical photo camera technique and allow a dynamic sequence review (cine loop).

Dynamic examinations are numerous in children: voiding cystourethrography, esophagography and upper gastro intestinal study, deglutition evaluations, dynamic laryngeal studies, and even angiography by subtraction is a possibility.

Digital fluorography has other very important advantages. Film economy is an advantage because it is possible to choose the best images to be reproduced and to perform a complete examination on a single film. This has a double advantage; the number of films is diminished and less space is required in overflowing files. The other advantages are its suitability for pediatric practice: radiation dose reduction and real-time study. The radiation dose reduction is a very important point in pediatrics. Digital fluorography can be expected to reduce the dose by approximately $25 \%$ to $50 \%$ compared with that required for $100-\mathrm{mm}$ spot film acquisition. However, two points must be emphasized. Firstly, the decrease in radiation dose varies

From the Pediatric Radiology Department, CHU de Grenoble, Grenoble, France.

Address reprint requests to $C h$. Durand, MD, Senvice de radio-pediatrie, Hopital de Grenoble BP217, 38043 Grenoble Cedex 9, France.

Copyright 11995 by W.B. Saunders Company

0897-1889/95/0801-1019\$3.00/0 
according to the field size and is less important with a 16-cm field. Secondly, the ease of utilization leads to a temptation to take additional films.

Real-time study allows immediate viewing of the images. This advantage is also important in pediatrics for several reasons. It allows important reduction of examination time and avoids unnecessary waiting for film development. The anxiety of the child (and of his parents) is thus reduced. Diagnostic efficacy is increased by the possibility of quickly checking diagnostic elements, which eliminates useless sequences. It also provides benefits in informing parents and training residents.

The biggest disadvantage of digital fluorography is lack of spatial resolution, which is 1.5 line pairs $/ \mathrm{mm}$ for a $30-\mathrm{cm}$ field. However, this may be limited. The use of small fields during the same examination partially compensate for the poor spatial resolution. Spatial resolution is improved, but the radiation dose is also increased.

\section{CONVENTIONAL RADIOGRAPHY}

During the first evaluations in patients with unknown pathology, evaluations require maximal spatial resolution. Digital fluorography is not sufficient and phosphostimulable plates are recommended for chest film, abdominal plain film, or bone radiology.

In some cases, digital fluorography may show principal lesions, but some pathologies may not be detected, such as small bronchi thickening, inter alveolar septa thickening, reticulo-micronodular opacities, abdominal micro calcifications, micro fractures, subperiosteal resorption images, or subtle periosteal appositions.

\section{CONTRAST STUDIES}

The lack of spatial resolution in digital fluorography is compensated by high-contrast resolution which allows a sufficient analysis of contrast studies such as intravenous pyelography, digestive opacifications, bronchography, arthrography, myelography, and direct opacifications. The only limitation is the field-of-view size in older children. In fact, analysis is sufficient, but image quality of phosphor plates is better. A choice between two methods is possible.

\section{SOME PARTICULAR SITUATIONS}

The use of photostimulable plates is mandatory for portable radiographs or in the operating room for technical reasons. Interventional radiology has the particularity of causing a high radiation dose and implies immediate utilization of the images. Digital fluorography is then suggested because it allows a real-time study. It is even possible to use fluorography, which suppressed all irradiation.

\section{SPECIFIC PEDIATRIC INDICATIONS}

Pediatric radiology is well adapted to digital fluorography because it allows a decrease in the radiation dose and reduces the child's and parent's stress and because the disadvantages that are noted in adults are less of a problem in children. We previously indicated that the field of view was limited to $30 \mathrm{~cm}$. This is a disadvantage in older children. In fact, in most pediatric cases, the child is small enough for visualization of the entire abdomen or thorax on a single view, and the small size of structures radiographed often allows the use of small fields of view, which improve spatial resolution. Theoretically, it is possible to use digital fluorography in numerous cases instead of plain films. The question is whether digital fluorography can be used for conventional radiography such as thoracic, abdominal, or osseous radiographs.

In fact, the true question is whether contrast resolution compensates for the lack of spatial resolution and is sufficient to assess a positive diagnosis. In our opinion, fluorography indications depends on two points: the necessity of obtaining maximal spatial resolution and the size of the organ to be analyzed. Spatial resolution is not always mandatory in some known situations such as tube control, bone age, fracture control, search for hip dysplasia, etc.

Sometimes the use of small fields of view is possible, either because the child is small or because the analyzed structure is small and spatial resolution may reach $2.5 \mathrm{lp} / \mathrm{mm}$. Some studies such as sinuses, sella, rhino pharynx, and hands are possible. However, the improvement of image quality is concomitant with an increase in the radiation dose that is directly proportional to decrease in field size. Under these conditions, dose-reduction benefits decrease and, in our experience, we prefer the use of 
phosphostimulable plates with better spatial resolution. In fact, the indication of one method compared with the other is a compromise between the necessity of obtaining good spatial resolution and the necessity of decreasing radiation dose in children. These are linked to the clinical information.

\section{CONCLUSION}

To summarize the main indications for each method, we conclude that digital fluorography is highly recommended for dynamic examinations, phosphor plates are highly recommended for conventional studies if the spatial resolution is mandatory, and either digital fluorography or phosphor plates can be used for contrast studies. In pediatric radiology, if the spatial resolution is not mandatory, digital fluorography is suggested to reduce the radiation dose and, if using a small field of view is possible, digital fluorography may be carefully discussed. Digital radiography is not supported to be better than classical radiology nor to compensate for technician's mistakes. It is aimed to improve the department's functioning and, above all, to accommodate future innovations. Images can be transferred or archived through networks. This is a main prospect in the coming years. 